\title{
Erratum to: Corrosion Behavior of Detonation Gun Sprayed Al Coating on Sintered NdFeB
}

Jizhao Ma, Xiaofang Liu, Wentao Qu, and Chungen Zhou

\section{Erratum to: Journal of Thermal Spray Technology \\ DOI 10.1007/s11666-014-0188-9}

We regret that the original article contained a typographical error in the title. The "d" was inadvertently left out of the element $\mathrm{Nd}$. The correct title should be
"Corrosion Behavior of Detonation Gun Sprayed Al Coating on Sintered NdFeB."

The online version of the original article can be found under doi:10.1007/s11666-014-0188-9.

Jizhao Ma, Xiaofang Liu, and Chungen Zhou, Department of Materials Science and Engineering, Beijing University of Aeronautics and Astronautics, Key Laboratory of Aerospace Materials and Performance (Ministry of Education), Beijing 100191, China; and Wentao Qu, School of Mechanical Engineering, Xi'an Shiyou University, Xi'an 710065, China. Contact e-mail: cgzhou@buaa.edu.cn. 